\title{
Accelerated removal of deamidated proteins and endogenous electric fields: possible implications
}

\author{
Isabella Panfoli, Silvia Ravera, Daniela Calzia and Alessandro Morelli \\ Department of Biology, University of Genoa, V.le Benedetto XV 3, 16132 Genova, Italy
}

\begin{abstract}
Changes in protein structure through the spontaneous deamidation of asparaginyl (Asn) and glutaminyl (Gln) residues have been observed in many proteins. Amide residues were supposed to serve as clocks for development and aging. Deamidated proteins are rapidly degraded by as yet unclear molecular mechanisms. Deamidation leads to elevation of the ratio of charged versus polar residues $(\mathrm{CH}-\mathrm{PO})$ of a protein and to a decrease in its $\mathrm{pI}$ value. We had reported that those enzymes, characterized by a high $\mathrm{CH}-\mathrm{PO}$, are prone to inactivation and loss of ordered structure by exposure to direct current from low voltage in solution. Nano-local endogenous electric fields arise within cells. Endogenous currents may cause the unfolding of the products of deamidation at Asn. In turn, these unfolded proteins would be removed, likely by proteolysis.
\end{abstract}

Key words: Aging - Asparagine - CH-PO - Deamidation - Direct electric current - Glutamine - Proteasome

Abbreviations: $\mathrm{CH}-\mathrm{PO}$, ratio of charged versus polar residues of a protein; $\mathrm{dc}$, direct electric current; Asn, asparagine; Gln, glutamine.

\section{Protein deamidation}

Many biological peptides and proteins possess labile asparaginyl (Asn) and glutaminyl (Gln) residues. Deamidation of Asn and Gln residues is now recognized as one of the major pathways of spontaneous protein degradation, together with protein aggregation, oxidation, and glycation (Robinson 2002; Reissner and Aswad 2003; Li et al. 2006).

The first isolation and characterization of the amidated and deamidated forms of a naturally occurring peptide was accomplished by counter-current distribution of insulin in 1952 (reviewed in Robinson 2004a). However, only about 40 years ago, it was discovered that deamidation occurs in vivo under genetic control, over a very wide, physiologically relevant deamidation rate range (Robinson et al. 1970). Subsequently, much has been learned, especially about Asn, whose deamidation rate is faster than Gln. Between 1971 and 1973, Robinson and co-workers examined the deamidation

Correspondence to: Isabella Panfoli, Department of Biology, University of Genova, V.le Benedetto XV 3, 16132 Genova, Italy E-mail: Isabella.Panfoli@unige.it of 65 synthetic peptides labeled with $\mathrm{C}^{14}$ and measured the deamidation rates at $\mathrm{pH} 7$, directly measuring the sequencedetermined deamidation rates of model peptides (reviewed in Robinson 2004a). It is now established that a large percentage of proteins deamidate to a substantial extent during their biological lifetimes.

The deamidation of Gln proceeds both enzymatically and non-enzymatically. By contrast, only the nonenzymatic deamidation of Asn has been reported. Deamidation rates seem to depend upon the sequence and higher-order structure of the protein and to affect protein structure and function in natural and engineered mutant sequences (Clarke 2003). In fact, since the side chains of Asn and Gln are not charged, deamidation introduces a negative charge at the site of occurrence, affecting the structure of the peptides and proteins in which they are incorporated. The occurrence of Asn and Gln in proteins was determined by Robinsons (2004b) from a set of 4835 representative proteins from the January 2003 PDB databank. Authors found that about $72 \%$ of proteins possessed more Asn and $63 \%$ have more Gln than expected by chance. The research literature on the history deamidation was reviewed and summarized (Robinson 2004a). 


\section{Reaction mechanisms of deamidation}

The reaction mechanisms of nonenzymatic deamidation have been extensively studied. In eukaryotes, the process of Asn deamidation is non-enzymatic, spontaneously occurring at neutral $\mathrm{pH}$. It proceeds primarily through a succinimide mechanism with also a small amount of direct hydrolysis. To date, enzymes involved in targeted deamidation of Asn in proteins have been only identified in prokaryotes (Horiguchi et al. 1997). Spontaneous Gln deamidation proceeds through a glutarimide, but, since this reaction is relatively slow, direct hydrolysis is more significant. The studies of Robinson et al. (2004a) on synthetic $\mathrm{C}^{14}$ labeled peptides showed that deamidation rates increased by His and Ser neighboring to Asn with the effect larger for the carboxyl-side residue are affected by steric hindrance from the side chains of the nearest neighboring residues to Asn and Gln and slowed by secondary and tertiary structure (Geiger and Clarke 1987; Robinson and Robinson 2004b). In addition, D-Asn and D-isoaspartyl residues can form (Reissner and Aswad 2003). Under physiological conditions, deamidation of Asn can occur with half-times as short as 6 h (Kim et al. 1999; Robinson and Robinson 2004b). Enzymes have been found that deamidate carboxyl terminal Asn (Kikuchi and Sakaguchi 1972) and amino terminal Asn (Balogh et al. 2000), however, these have no ability to deamidate the side chains of Asn. Asparaginases, acting on free asparagine, are also known.

The enzymatic deamidation of Gln residues by transglutaminases, proteases, and glutaminases, is widely distributed from microorganisms to mammals and is involved in several ordinary and pathological processes. For example, tissue transglutaminase (TG2), a calcium-dependent multifunctional enzyme, produces Gln deamidation as a side reaction that can modify proteins by transamidation or deamidation of specific Gln residues (Lorand 2002). TG2 deamidating activity seems to play a major role in the pathogenesis of celiac disease, as it generates deamidated gliadin peptides that are recognized by $\mathrm{CD}^{+} \mathrm{T}$ cells (Mazzeo et al. 2003).

\section{Pivotal role of deamidation in the regulation of biologi- cal properties}

It seems that the physiological significance of deamidation lays in the alterations of tertiary structure of a protein it produces. For example, deamidation of triose phosphate isomerase (P00939, ExPASy) decreases subunit-subunit interactions, exposing the hydrophobic interface to the aqueous environment (Sun et al. 1995). Deletion experiments showed that deamidation of two Asn of $\mathrm{Bcl}-\mathrm{x}_{\mathrm{L}}$ disrupt its antiapoptotic activity, as the negatively charged deamida- tion products alter $\mathrm{Bcl}-\mathrm{x}_{\mathrm{L}}$ tertiary structure (Deverman et al. 2002). The glial myelin basic protein (MBP) (P02686, Expasy) undergoes deamidation of Gln producing Glu as well as of Arg, producing citrulline (Coenen et al. 2007), which decreases its cationic properties, in turn affecting adhesion to the two cytosolic layers of myelin sheath (Min et al. 2009). Differential deamidation regulates cellular and developmental processes ( $\mathrm{Li}$ and Thompson 2002). Moreover, deamidation leads to faster degradation in vivo (Robinson and Robinson 2004a). However, the knowledge of critical passages of the removal of the deamidated proteins is still limited.

A computation procedure for quantitatively combined primary-structure determined deamidation rate and the higher order structure modulation of that rate has been developed for 170,014 Asn residues in 13,335 proteins (Robinson 2002; http://www.deamidation.entrewave. com). The computed rates show that significant amounts of deamidation may be expected to occur in a substantial percentage of proteins under physiological conditions. It is now established, therefore, that a large percentage of proteins deamidate to a substantial extent during their biological lifetimes. Deamidation can control in vivo protein turnover. The protein pool is a dynamic, time-dependent array of protein molecules, whose structures are continuously changing under the control of miniature amide clocks imbedded in each protein (Robinson 2002). The genetically specified primary sequence control of nonenzymatic deamidation of Asn and Gln under physiological conditions provides a range of deamidation half-times between about 1 day and 50 years. The combination of multiple amides in proteins and the modulation of deamidation by secondary, tertiary, and quaternary structure provide a currently known range of physiological protein deamidation half-times between a few hours and more than a century.

\section{Deamidation and aging}

The hypothesis that deamidation may serve as a molecular clock in aging of organism was first published by Robinson, McKerrow, and Cary in 1970 (Robinson et al. 1970). Thereafter, this hypothesis became known as the "deamidation theory of aging" and took its place among the many other biochemical processes that have been proposed as the mechanisms of aging (Moffat 1980). Analysis of protein sequence suggests that Asn and Gln ability has been positively selected in the evolution of proteins, possibly playing a role in the regulation of time-dependent biological processes such as protein breakdown, development and aging (Robinson and Robinson 2004a). Also, a correlation was found between the percentage of amide residues, and the 
in vivo turnover rate in 12 proteins. Deamidation would be a molecular clock, determining the mean lifetime of certain proteins in vivo. If Asn serves as a ubiquitous molecular clock, a lack of natural asparaginases is expected, in fact they have never been described. This fact reflects a difference between Asn and Gln in their usefulness as biomolecular clocks.

The hypothesis that nonenzymatic deamidation serves as an ubiquitous molecular clock for the regulation of biological processes has been strengthened by studies showing that nonenzymatic deamidation controls the in vivo turnover rates of cytochrome $c$ and aldolase. Deamidation of cytochrome $c$ leads to its rapid catabolism in vivo, with rates equivalent to the turnover rate (Robinson 2004b). Similarly, the deamidation of the aldolase C-terminal sequence and the in vivo turnover proceed at the same rates (Robinson and Robinson 2004b).

\section{$\mathrm{CH}-\mathrm{PO}$ parameter}

A lot of thermostable proteins display a large difference between charged (Asp, Glu, Lys, Arg) versus polar (Asn, Gln, Ser, Thr) amino acids (Suhre and Claverie 2003). CH-PO value for hyperthermophilic proteins is higher $(10 \div 15.0)$ in comparison with mesophilic $(-5.0 \div 5.0)$ ones (Suhre and Claverie 2003). Polar residues, tending to introduce the aqueous solvent into the core of the protein, are less represented in hyperthermophilic proteins, that are stabilized by a network of ion bonds at the protein surface (Kumar and Nussinov 2001). A few mesophyle enzymes still display high $\mathrm{CH}-\mathrm{PO}$ (e.g. 11.37 for human DNA polymerase catalytic subunit; 25.8 for human DNA topoisomerase), and may be vestigial of our ancestors dwelling in drastic environmental conditions. On the other hand, other factors contribute toward thermal stability of other thermostable proteins (i.e. increase in secondary structure, hydrophobic interactions, disulphide bonds and oligomerization) (Sakuraba et al. 2003; Yano and Poulos 2003).

\section{Endogenous direct currents}

Extra- and intra-cellular electrical currents arise in vivo, as a result of the diffusion and convection of charged species, spanning a broad frequency range, from the milliHertz of injury currents up to hundreds of Hertz around excitable membranes (reviewed in McCaig et al. 2005). Currents of $100 \mu \mathrm{A} / \mathrm{cm}^{2}$ have been measured at the blastopore of Xenopus embryos. There is growing evidence that endogenous electric fields (EF), and consequent endogenous dc, are present at wounds and in areas of active cell growth and migration during development. In rat cornea wound healing seems regulated by the wound-induced EF (McCaig et al. 2005). Endogenous EF are essential to regulate cell behaviour during tissue morphogenesis and regeneration (Song et al. 2004; Saunders and McCaig 2005). It would be interesting to know whether there are variations in protein deamidation in the development and wound healing processes. It was reported that the Escherichia coli cytotoxic necrotizing factor 1, which catalyzes the deamidation of the Rho GTP-binding proteins at a specific Gln, impairs intestinal epithelial wound healing (Brest et al. 2004). Also, TG2 affects cell-matrix interactions in cell spreading, migration and extracellular matrix reorganization in wound healing (Hasan et al. 2006). However, in the latter two cases deamidation is not spontaneous.

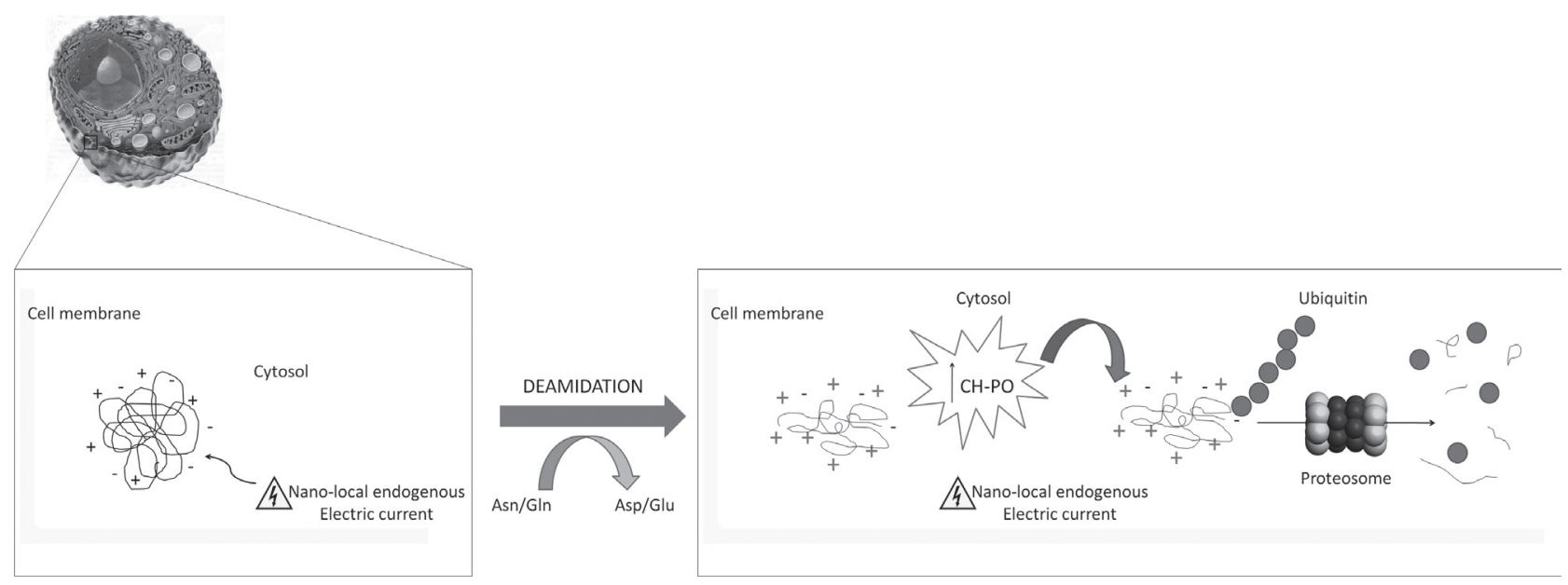

Figure 1. Hypothetic route for the degradation of deamidated proteins. It is supposed that proteins undergoing an increase in $\mathrm{CH}-\mathrm{PO}$ over a threshold value of $(10 \pm 1.4)$ and a decrease in $\mathrm{pI}$ upon spontaneous deamidation would be prone to permanent unfolding by nanolocal direct-current electric fields, recognized as damaged and sent to proteasomal degradation. 
Table 1. Measured versus theoretically calculated $p I$ values for 12 proteins in the paper from Dice and Goldberg (1975)

\begin{tabular}{lcccc}
\hline Protein & Swiss Prot & Total AA number & Measured pI & Calculated pI \\
\hline S-amino levulinate synthetase & P06214 & 330 & 5.9 & 6.32 \\
Aspartate amino transferase & P00507 & 430 & 5.6 & 9.13 \\
Catalase & P04762 & 526 & 6.7 & 7.07 \\
Ferritin & P19132 & 181 & 5.7 & 5.86 \\
Glucose-6-phosphate dehydrogenase & P05370 & 514 & 5.3 & 5.97 \\
Histidase & P21213 & 657 & 5.4 & 6.07 \\
Ornithine amino transferase & P04182 & 439 & 5.4 & 6.53 \\
Ornithine decarboxylase & P09057 & 461 & 4.1 & 5.21 \\
Phosphoenolpyruvate carboxylase & P07379 & 622 & 5.0 & 6.09 \\
Pyruvate kinase & P11980 & 530 & 6.0 & 6.69 \\
Tryptophan oxygenase & P09810 & 444 & 5.0 & 6.3 \\
Tyrosine amino transferase & P04694 & 454 & 4.1 & 5.27 \\
\hline
\end{tabular}

Swiss Prot, ExPASy proteomics server accession number (www.expasy.org); total AA number, number of aminoacids (AA); measured pI, value measured by the author; calculated pI, value theoretically calculated according to ExPaSy.

\section{Interaction of $\mathrm{dc}$ and proteins with elevated $\mathrm{CH}-\mathrm{PO}$}

Interaction between electric current and proteins is a little explored topic. We reported that exposure of Crotalus atrox venom to dc in solution caused inactivation of venom phospholipase $\mathrm{A}_{2}$ and metalloproteases (Panfoli et al. 2007). We also reported that enzymes with $\mathrm{CH}-\mathrm{PO}$ higher than 10.0 are irreversibly inactivated by $\mathrm{dc}$ exposure (in the range
0-0.7 mA) from low voltage $(0-10 \mathrm{~V})$. By contrast, those with $\mathrm{CH}-\mathrm{PO}$ in the range -5 and +3 are not (Calzia et al. 2009). Inactivation was dependent on the ionic strength of the medium and not on the net charge of the protein. The effect seemed to be due to the current density. Some of the inactivated enzymes underwent a structural modification measurable by circular dichroic spectroscopy. It was concluded that $\mathrm{CH}-\mathrm{PO}$ can predict protein inactivation by dc exposure (Calzia et al. 2009). Interestingly, as stated

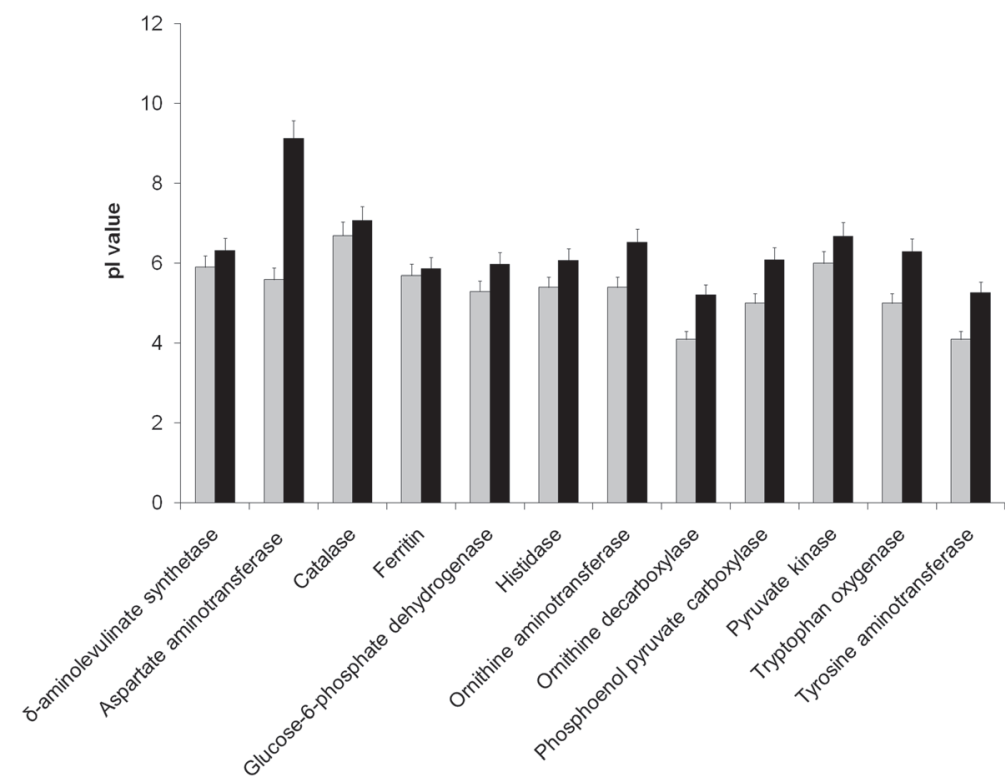

Figure 2. Theoretical versus measured pI values for 12 proteins selected from Table 1 in the paper Dice and Goldberg (1975). Figure reports pI value, as measured by the authors (grey columns), or pI value theoretically calculated (black columns) according to ExPaSy (www.expasy.org). 
Table 2. Estimated CH-PO change for some of the enzymes reported in Table 1, after spontaneous deamidation

\begin{tabular}{|c|c|c|c|c|c|c|c|}
\hline Protein & $\begin{array}{l}\text { PDB } \\
\text { code }\end{array}$ & Total AA & KRDE & STQN & $\mathrm{CH}-\mathrm{PO}$ & $\begin{array}{c}\text { deamidable } \\
\text { Asn }\end{array}$ & $\begin{array}{c}\mathrm{CH}-\mathrm{PO} \\
\text { (deamid.) }\end{array}$ \\
\hline Aldolase & $1 \mathrm{ADO}$ & 248 & 58 & 46 & 4.8 & 1 & 5.64 \\
\hline Calbindin & $4 \mathrm{ICB}$ & 79 & 28 & 15 & 16.45 & 1 & 18.99 \\
\hline Cytochrome $c$ & $2 \mathrm{GIW}$ & 105 & 33 & 18 & 14.28 & 2 & 18.09 \\
\hline Fatty acid-binding protein & $1 \mathrm{LFO}$ & 127 & 37 & 28 & 7.09 & 1 & 8.66 \\
\hline Fibroblast growth factor & $2 \mathrm{AFG}$ & 140 & 36 & 29 & 5 & 1 & 6.43 \\
\hline Hemoglobin & $1 \mathrm{~A} 3 \mathrm{~N}$ & 142 & 26 & 25 & 0.70 & 7 & 10.56 \\
\hline $\begin{array}{l}\text { Hypoxanthine guanine phos- } \\
\text { phoribosyl transferase }\end{array}$ & $1 \mathrm{BZY}$ & 217 & 58 & 33 & 11.5 & 1 & 12.44 \\
\hline Triose phosphate Isomerase & $1 \mathrm{HTI}$ & 248 & 62 & 42 & 8.06 & 2 & 9.67 \\
\hline
\end{tabular}

Table reports data for 8 proteins selected from Table 1: PDB code (www.rcb.org); total number of aminoacids (AA); content in charged (KRDE) and polar (STQN) AA; CH-PO value; number of deamidable Asn reported in Robinson web site (www.deamidation.org); change in $\mathrm{CH}-\mathrm{PO}$ after deamidation of deamidable Asn.

above, $\mathrm{CH}-\mathrm{PO}$ of deamidated proteins increases. In fact, a polar AA (Asn or Gln) turns into a charged AA (Asp or $\mathrm{Glu})$. Considering that an elevated $\mathrm{CH}-\mathrm{PO}$ value renders a protein prone to inactivation by dc exposure (Calzia et al. 2009), and that this electro-inactivation is often accompanied by a change in tertiary structure, it may be supposed that the more highly charged products of deamidation at Asn become sensitive to endogenous currents (Calzia et al. 2009). These currents may produce an "electro-stretch" and unfolding of the deamidated protein (Calzia et al. 2009) which is eventually proteolysed (Fig. 1). A degradation of the unfolded proteins may explain why Robinson et al. did not find the expected amount of deamidated proteins within the cells (Robinson 2002).

\section{Do deamidated misfolded proteins undergo proteolysis?}

An inverse correlation was found between protein Asn + Gln content and their stability (Rogers and Rechsteiner 1988). Deficiency of the repair cytosolic enzyme L-isoaspartate (D-aspartate) O-methyltransferase (PIMT, EC 2.1.1.77),

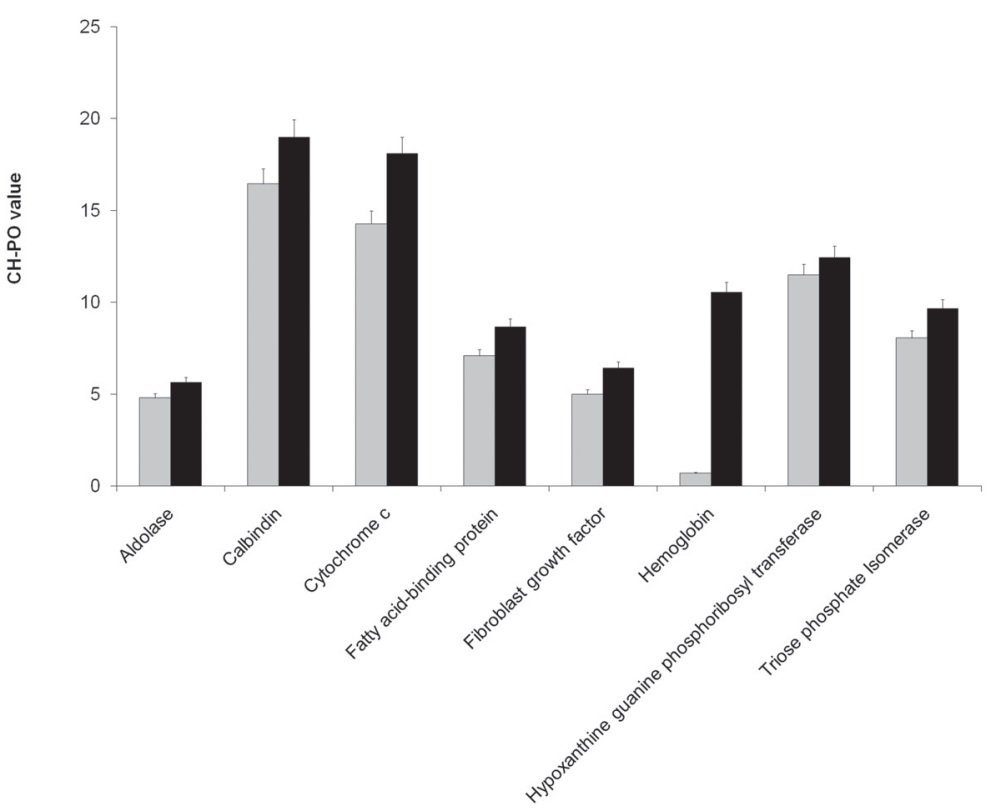

Figure 3. Estimated change in $\mathrm{CH}-\mathrm{PO}$ of some enzymes after spontaneous deamidation. Figure reports $\mathrm{CH}-\mathrm{PO}$ value (grey columns) and change in CH-PO after Asn deamidation (black columns) of some proteins used in the paper Robinson (2002). 
that converts altered aspartyl residues to normal L-aspartyl residues (Ryttersgaard et al. 2002), results in the accumulation of altered proteins with fatal consequences in knockout mice (Kim et al. 1999). PMIT cannot restore the amide group to Asn residues (Ota and Clarke 1989), but converts the isopeptide to a normal one, that can be hydrolyzed (Galletti et al. 1995). By contrast, an extension of life up to $30 \%$ for Drosphilia overexpressing PIMT was reported (Chavous et al. 2001).

Studies from Dice and Goldberg (1975) on protein turnover in vivo showed that acidic proteins are degraded faster. Figure 2 shows that the pI values measured by electrophoresis of some of the proteins in the paper by Dice and Goldberg (1975) appear lower than the theoretic ones, calculated according to ExPaSy Proteomic Server (see Table 1), as though the proteins were already deamidated in vivo. The proteins for which the authors measured the most elevated turnover rates were those possessing the lower pI values. Deamidated proteins become more acidic at physiological $\mathrm{pH}$, and their $\mathrm{CH}-\mathrm{PO}$ rises. Interestingly, $\mathrm{CH}-\mathrm{PO}$ values of the proteins that displayed elevated turnover rates were over 5.

Figure 3 shows that the $\mathrm{CH}-\mathrm{PO}$ value of some proteins reported in Robinson (2002) always rises over the mean value for the mesophyle proteins after deamidation of the deamidable Asn. The number of deamidable Asn for each protein was obtained by a query with the PDB identifier in the CD and ID Database (www.deamidation.org). Table 2 shows data that served to build Fig. 2 .

May be one cause of cell ageing the removal of some spontaneously deamidated proteins after irreversible loss of ordered structure? Elucidation of these aspects may be an essential step towards an understanding of key biological processes.

\section{References}

Balogh S. A., Kwon Y. T., Denenberg V. H. (2000): Varying intertrial interval reveals temporally defined memory deficits and enhancements in NTAN1-deficient mice. Learn. Mem. 7, 279-286; doi:10.1101/lm.33500

Brest P., Turchi L., Le'Negrate G., Berto F., Moreilhon C., Mari B., Ponzio G., Hofman P. (2004): Escherichia coli cytotoxic necrotizing factor 1 inhibits intestinal epithelial wound healing in vitro after mechanical injury. Infect. Immun. 72, 5733-5740; doi:10.1128/IAI.72.10.5733-5740.2004

Calzia D., Panfoli I., Ravera S., Dazzi E., Gandolfo S., Pepe I. M., Vergani L., Morelli A. M. (2009): Structural modification of proteins by direct electric current from low voltage. J. Biochem. Mol. Toxicol. 23, 309-317; doi:10.1002/jbt.20293

Chavous D. A., Jackson F. R., O'Connor C. M. (2001): Extension of the Drosophila lifespan by overexpression of a protein repair methyltransferase. Proc. Natl. Acad. Sci. U. S. A. 98, 14814-14818; doi:10.1073/pnas.251446498

Clarke S. (2003): Aging as war between chemical and biochemical processes: protein methylation and the recognition of age- damaged proteins for repair. Ageing Res. Rev. 2, 263-285; doi:10.1016/S1568-1637(03)00011-4

Coenen D., Verschueren P., Westhovens R., Bossuyt X. (2007): Technical and diagnostic performance of 6 assays for the measurement of citrullinated protein/peptide antibodies in the diagnosis of rheumatoid arthritis. Clin. Chem. 53, 498-504; doi:10.1373/clinchem.2006.078063

Deverman B. E., Cook B. L., Manson S. R., Niederhoff R. A., Langer E. M., Rosova I., Kulans L. A., Fu X., Weinberg J. S., Heinecke J. W., Roth K. A., Weintraub S. J. (2002): Bcl-xL deamidation is a critical switch in the regulation of the response to DNA damage. Cell 111, 51-62

Dice J. F., Goldberg A. L. (1975): Relationship between in vivo degradative rates and isoelectric points of proteins. Proc. Natl. Acad. Sci. U. S. A. 72, 3893-3897; doi:10.1073/ pnas.72.10.3893

Galletti P., Ingrosso D., Manna C., Clemente G., Zappia V. (1995): Protein damage and methylation-mediated repair in the erythrocyte. Biochem. J. 306, 313-325

Geiger T., Clarke S. (1987): Deamidation, isomerization, and racemization at asparaginyl and aspartyl residues in peptides. Succinimide-linked reactions that contribute to protein degradation. J. Biol. Chem. 262, 785-794

Hasan Q., Alluri R. V., Rao P., Ahuja Y. R. (2006): Role of glutamine deamidation in neurodegenerative diseases associated with triplet repeat expansions: a hypothesis. J. Mol. Neurosci. 29, 29-33; doi:10.1385/JMN:29:1:29

Horiguchi Y., Inoue N., Masuda M., Kashimoto T., Katahira J., Sugimoto N., Matsuda M. (1997): Bordetella bronchiseptica dermonecrotizing toxin induces reorganization of actin stress fibers through deamidation of Gln-63 of the GTPbinding protein Rho. Proc. Natl. Acad. Sci. U. S. A. 94, 11623-11626; doi:10.1073/pnas.94.21.11623

Kikuchi M., Sakaguchi K. (1972): Peptidoasparaginase. An enzyme for deamidation of $\mathrm{COOH}$-terminal peptide-bound asparagine. Arch. Biochem. Biophys. 148, 315-317; doi:10.1016/0003-9861(72)90146-4

Kim E., Lowenson J. D., Clarke S., Young S. G. (1999): Phenotypic analysis of seizure-prone mice lacking L-isoaspartate (D-aspartate) O-methyltransferase. J. Biol. Chem. 274, 20671-20678; doi:10.1074/jbc.274.29.20671

Kumar S., Nussinov R. (2001): How do thermophilic proteins deal with heat? Cell. Mol. Life Sci. 58, 1216-1233; doi:10.1007/ PL00000935

Li B., Schowen R. L., Topp E. M., Borchardt R. T. (2006): Effect of $\mathrm{N}-1$ and $\mathrm{N}-2$ residues on peptide deamidation rate in solution and solid state. APS J. 8, E166-173

Li C., Thompson C. B. (2002): Cancer. DNA damage, deamidation, and death. Science 298, 1346-1347; doi:10.1126/science. 1079168

Lorand L. (2002): Transglutaminase: remembering Heinrich Waelsch. Neurochem. Int. 40, 7-12; doi:10.1016/S01970186(01)00056-0

Mazzeo M. F., De Giulio B., Senger S., Rossi M., Malorni A., Siciliano R. A. (2003): Identification of transglutaminase-mediated deamidation sites in a recombinant alpha-gliadin by advanced mass-spectrometric methodologies. Protein Sci. 12, 2434-2442; doi:10.1110/ps.03185903 
McCaig C. D., Rajnicek A. M., Song B., Zhao M. (2005): Controlling cell behavior electrically: current views and future potential. Physiol. Rev. 85, 943-978; doi:10.1152/physrev.00020.2004

Min Y., Kristiansen K., Boggs J. M., Husted C., Zasadzinski J. A., Israelachvili J. (2009): Interaction forces and adhesion of supported myelin lipid bilayers modulated by myelin basic protein. Proc. Natl. Acad. Sci. U. S. A. 106, 3154-3159; doi:10.1073/pnas.0813110106

Moffat K. (1980): Crystallization of the peptide hormone, human chorionic somatomammotropin. Int. J. Pept. Protein Res. 15, 149-153

Ota I. M., Clarke S. (1989): Enzymatic methylation of L-isoaspartyl residues derived from aspartyl residues in affinity-purified calmodulin. The role of conformational flexibility in spontaneous isoaspartyl formation. J. Biol. Chem. 264, 54-60

Panfoli I., Ravera S., Calzia D., Dazzi E., Gandolfo S., Pepe I. M., Morelli A. (2007): Inactivation of phospholipase A(2) and metalloproteinase from Crotalus atrox venom by direct current. J. Biochem. Mol. Toxicol. 21, 7-12; doi:10.1002/ jbt. 20152

Reissner K. J., Aswad D. W. (2003): Deamidation and isoaspartate formation in proteins: unwanted alterations or surreptitious signals? Cell. Mol. Life Sci. 60, 1281-95; doi:10.1007/ s00018-003-2287-5

Robinson A. B., McKerrow J. H., Cary P. (1970): Controlled deamidation of peptides and proteins: an experimental hazard and a possible biological timer. Proc. Natl. Acad. Sci. U. S. A. 66, 753-757; doi:10.1073/pnas.66.3.753

Robinson N. E., (2002): Protein deamidation. Proc. Natl. Acad. Sci. U. S. A. 99, 5283-5288; doi:10.1073/pnas.082102799

Robinson N. E., Robinson A. B. (2004a): Molecular Clocks: Deamidation of Asparaginyl and Glutaminyl Residues in Peptides and Proteins. Althouse Press, Cave Junction, OR.

Robinson N. E., Robinson A. B. (2004b): Amide molecular clocks in drosophila proteins: potential regulators of aging and other processes. Mech. Ageing Dev. 125, 259-267; doi:10.1016/j.mad.2004.01.001

Rogers S. W., Rechsteiner M. (1988): Degradation of structurally characterized proteins injected into HeLa cells. Tests of hypotheses. J. Biol. Chem. 263, 19850-19862

Ryttersgaard C., Griffith S. C., Sawaya M. R., MacLaren D. C., Clarke S., Yeates T. O. (2002): Crystal structure of human L-isoaspartyl methyltransferase. J. Biol. Chem. 277, 10642-10646; doi:10.1074/jbc.M200229200

Sakuraba H., Tsuge H., Shimoya I. Kawakami R., Goda S., Kawarabayasi Y., Katunuma N., Ago H., Miyano M., Ohshima T. (2003): The first crystal structure of archaeal aldolase. Unique tetrameric structure of 2-deoxy-d-ribose-5-phosphate aldolase from the hyperthermophilic archaea Aeropyrum pernix. J. Biol. Chem. 278, 10799-10806; doi:10.1074/jbc.M212449200

Saunders R. D., McCaig C. D. (2005): Developmental effects of physiologically weak electric fields and heat: an overview. Bioelectromagnetics Suppl. 7, S127-132; doi:10.1002/ bem. 20117

Song B., Zhao M., Forrester J., McCaig C. (2004): Nerve regeneration and wound healing are stimulated and directed by an endogenous electrical field in vivo. J. Cell Sci. 117, 4681-4690; doi:10.1242/jcs.01341

Suhre K., Claverie J. M. (2003): Genomic correlates of hyperthermostability, an update. J. Biol. Chem. 278, 17198-17202; doi:10.1074/jbc.M301327200

Sun A. Q., Yuksel K. U., Gracy R. W. (1995): Terminal marking of triosephosphate isomerase: consequences of deamidation. Arch. Biochem. Biophys. 322, 361-368; doi:10.1006/abbi.1995.1476

Yano J. K., Poulos T. L. (2003): New understandings of thermostable and peizostable enzymes. Curr. Opin. Biotechnol. 14, 360-365; doi:10.1016/S0958-1669(03)00075-2

Received: December 2, 2009

Final version accepted: March 31, 2010 